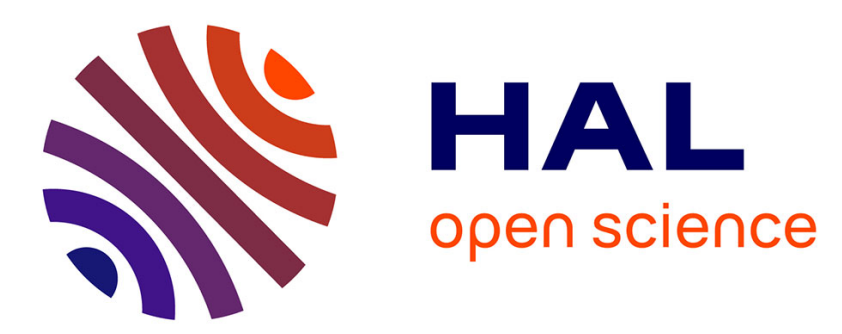

\title{
Interactions between Topographic Irregularities and Seismic Ground Motion Investigated Using a Hybrid FD-FE Method
}

\author{
Ariane Ducellier, Hideo Aochi
}

\section{> To cite this version:}

Ariane Ducellier, Hideo Aochi. Interactions between Topographic Irregularities and Seismic Ground Motion Investigated Using a Hybrid FD-FE Method. Bulletin of Earthquake Engineering, 2012, 10 (3), pp.773-792. 10.1007/s10518-011-9335-6 . hal-00653341

\section{HAL Id: hal-00653341 https: / hal-brgm.archives-ouvertes.fr/hal-00653341}

Submitted on 19 Dec 2011

HAL is a multi-disciplinary open access archive for the deposit and dissemination of scientific research documents, whether they are published or not. The documents may come from teaching and research institutions in France or abroad, or from public or private research centers.
L'archive ouverte pluridisciplinaire HAL, est destinée au dépôt et à la diffusion de documents scientifiques de niveau recherche, publiés ou non, émanant des établissements d'enseignement et de recherche français ou étrangers, des laboratoires publics ou privés. 


\section{Interactions between Topographic Irregularities and Seismic Ground Motion Investigated Using a Hybrid FD-FE Method}

Ariane Ducellier, Hideo Aochi

BRGM, 3 avenue Claude Guillemin, BP 36009, 45060 Orléans CEDEX 2, France

Phone: + 33238644891

Fax: + 33238644738

e-mail: a.ducellier@brgm.fr

Web site: http://www.brgm.fr

Abstract: A hybrid method combining finite element and $4^{\text {th }}$-order finite difference techniques is developed to model SH and P-SV seismic wave propagation in a 2D elastic medium with irregular surface topography. Both the classic staggered grid finite difference scheme and the partially staggered grid scheme are tested. The accuracy of the hybrid method is studied by comparison with a semi-analytical and another numerical method. Subsequently, to study the amplification, numerical simulations of seismic wave propagation in a series of hills are carried out and compared with the single-hill case. Depending on the position of the source in relation to the topography, the ratio between the heights and lengths of the hills or the ratio between the lengths of the hills and the wavelength, the presence of several hills as opposed to a single one can increase the amplification effect due to topography. This study highlights the fact that, when evaluating topographic site effects, surrounding topography must be taken into account in addition to local topography.

Keywords: Finite difference, Finite element, Hybrid methods, Seismic wave propagation, Topographic effects 


\section{Introduction}

Simulating ground motion at given sites is an essential task in the framework of seismic hazard studies. In most practical cases the source-site distance is large enough and local conditions are fairly simple from a geometrical standpoint; soil behaviour is therefore simulated in a 1D elastic/anelastic column under seismic excitation at the base of the column. However, some cases are much more complex, precluding a $1 \mathrm{D}$ approximation. It is known that a $2 \mathrm{D}$ or even 3D structure close to the site considered can significantly affect ground motion (e.g. Griffiths and Bollinger 1979, Hartzell et al. 1994, Spudich et al. 1996). Furthermore, the characteristics of the incident wave also become important factors when the source-site distance is short. To deal with a $2 \mathrm{D}$ (and 3D) context, numerical simulations are needed, since the characteristic ground motion features vary on a case by case basis, and it is accordingly difficult to quantify the effect empirically.

Ground motion can be simulated using volumetric methods such as those involving finite differences or finite elements. One of the most popular methods for simulating the seismic wave propagation in an elastic medium numerically is the finite difference method (FDM) (e.g. Madariaga 1976; Levander 1988) due to its simplicity, efficiency and ease of implementation. However, it is not capable of simulating complex boundary conditions like surface topography numerically (Ohminato and Chouet 1997). This is quite inconvenient, for taking into account sedimentary basins with strongly contrasted impedances or irregular topography is extremely useful when studying site effects for seismic hazard evaluation. As the finite difference method generally uses a uniform grid, accurate modelling of sedimentary layers near the surface with slow wave velocities involves a dramatic 
increase in the computational dimensions of the model in a large majority of instances (see Aoi and Fujiwara 1999 for an exception). An alternative method is the finite element method (FEM). It allows the use of irregular grids with elements of different sizes and geometries and is therefore quite useful to simulate complex geometry and to model strong heterogeneities in the velocity structure. However, this method requires significantly more memory than the finite difference method. A derivative version of the finite element method, of higher order, called the spectral element method (SEM) (Komatitsch and Vilotte 1998), has been developed and is very accurate and faster than any classic finite element method. Nonetheless, the finite difference method is still more attractive from the standpoint of the required computing resource, and it seems difficult to implant in the SEM the non-linear dynamics required in soil dynamics.

Various combinations of numerical methods for simulating seismic wave propagation in an elastic/anelastic medium were proposed in a large number of studies: hybrid discrete wave-number - finite difference method (Fäh 1992; Zahradník and Moczo 1996), hybrid boundary element - finite element method (Bielak et al. 2003; Gatmiri et al. 2008), and many others. Such a combination confers advantages not provided by a single method on its own. In particular, the combination of finite difference and finite element methods is expected to retain the efficiency of the finite difference method at large scale and to introduce the geometrical flexibility of the finite element method locally. Therefore, in this paper, we try to adapt the hybrid method previously proposed by Moczo et al. (1997) and Ma et al. (2004), to study ground motion resulting from irregular surface topography. We use various finite element and finite difference methods to simulate wave propagation in a $2 \mathrm{D}$ elastic medium. The finite difference method makes it possible to model both a point source and an extended fault: we 
thus will be able to model the effects of the topography on the wave generated by a complex source. Previously, De Martin et al. (2007) were able to simulate the wave propagation sequentially from the FDM to FEM domains by transferring the wave field information along the boundary. This is a practical and convenient way to simulate ground motion locally for the site considered. However, it should be noted that the local wavefield in the FEM does not impact the regional wavefield in the FDM. This assumption and scheme are accurate enough in many cases; however, in this study, we highlight cases where local disturbance in the wavefield is also important at the regional scale. This is why we need a complete hybrid simulation technique. Many earlier studies have numerically modelled seismic wave scatter around a single canyon or hill (e.g. Gaffet and Bouchon 1989, and many others), mainly in order to compare the results of numerical approaches with an analytical or semi-analytical solution. Also, some authors have applied numerical methods to study wave propagation in more complex real cases. However, few systematic studies have been conducted to determine the effects on ground motion of the interaction of a series of irregular topographies. To do so, we need to understand how the surface irregularities interact with each other. In the following sections, we begin by verifying our numerical approach and continue with several parametric studies. We will discuss how topographic amplification and resonance phenomena appear in various topographical settings.

\section{The numerical method}

In this section, we briefly explain the numerical approach of the hybrid finite difference - finite element method following Ma et al. (2004). The computational domain is divided into three sub-computational areas: one where only a standard finite difference method (FDM) is used to solve the seismic wave 
equation, a second where only an explicit finite element method (FEM) is used, and a third interface area where both FDM and FEM are used and the finite element mesh is made up of regular square meshes to facilitate interpolation of the wave field between the FD and the FE schemes. Examples of the domain partition are shown in Figures 1a and b.

Ma et al. (2004) used the standard staggered finite difference grid (Madariaga 1976; Virieux 1986). As collocation points (stress and velocity) are different, i.e., shifted, in each scheme, the velocity field is assumed to be a $3^{\text {rd }}$ order polynomial function and interpolated. This negatively impacts accuracy, but the use of higher-order interpolations might cause instabilities (Ma et al. 2004). This is a weak point in this hybrid method. Although Badea et al. (2008) do not specify what their finite difference method entails, their previous work (Wolf et al. 2006) tested different schemes, including a partially staggered finite difference grid (Saenger et al. 2000). The advantage of this latter grid scheme is that both horizontal and vertical velocities are computed for the same place, so no interpolation is needed. Here, we have adopted both finite difference schemes. As to the finite element scheme, we have tested higher order meshes, that is, spectral elements as well. However, this did not work in the trials we conducted. Indeed, the use of spectral elements results in high-order interpolation schemes in the interface between FDM and SEM areas. We believe that this does not ensure stability in the computation, as was the case in the study by Ma et al. (2004).

The influence of boundary conditions on the results of the computation is well known. For all the model boundaries excepting the free surface, where only finite differences are used, we introduce a convolutional PML (Komatitch and Martin 2007) to avoid artificial reflections. On the free surface, both finite difference and finite element methods are used. In the FD part, we use a stress- 
imaging method (Graves 1996) to model the free surface. In the FE part, the free surface condition is obtained by imposing a zero value of stress in the variational formulation.

\section{Numerical verification for simple cases}

Each part of the hybrid method was numerically verified for various wave propagation settings. Now we need to verify the hybrid method for some simple cases by comparison with analytical solutions and another numerical method.

Figures 2 and 3 show classic examples where the plane wave propagates upward in a homogeneous elastic medium towards a semi-circular canyon of radius $a$ (model geometry shown in Figure 1) for which the solution is (semi-) analytically given by an eigenfunction expansion method (Trifunac $(1971 ; 1973)$ for a SH problem and Wong $(1979 ; 1982)$ for a P-SV problem) and by a wavenumber method (Kawase 1988) for both cases. We suppose an incident wave in the FDM domain with a second-order Ricker function:

$$
u(t)=2\left(0.5-\left(\frac{\pi\left(t-t_{s}\right)}{t_{p}}\right)^{2}\right) \exp \left(-\left(\frac{\pi\left(t-t_{s}\right)}{t_{p}}\right)^{2}\right)
$$

where the characteristic angular frequency is given by $\omega_{0}=2 \pi / t_{p}$, and for the calculation we take $\omega_{0}=(2 \pi \beta) / a$, where $\beta$ is the shear wave velocity. The Poisson ratio is taken as $1 / 3$ for the P-SV problem. The size of the finite difference grid spacing is $0.05 * a$. The size of the irregular finite element meshes is approximately equal to the finite difference grid spacing (see Figures $1 \mathrm{a}$ and $\mathrm{b}$ ). The time step is taken as $0.0005 *(a / \beta)$. Figures 2 and 3 represent the $\mathrm{SH}$ and $\mathrm{P}$ SV cases respectively, showing the displacement amplification at main frequency $f_{0}=\omega_{0} / 2 \pi$ along the ground surface. Along the abscissa we have plotted the 
distance from the centre of the canyon, normalized by the canyon radius $a$. On the ordinate axis is the displacement amplification.

Figure 2 shows the case of a null wave angle of incidence (top panels) with the fully staggered grid (FSG) finite difference scheme (left panel) and the partially staggered grid (PSG) finite difference scheme (right panel), and the case of a $30^{\circ}$ anticlockwise wave angle of incidence (bottom panels) for the FSG (left panel) and PSG (right panel) cases. The line shows the numerical results obtained with the hybrid method, while the points represent the results with the (semi-) analytical method taken from Kawase (1988). Figure 3 shows the case of a horizontal incident wave (top panels) and a vertical incident wave (bottom panels) with a null wave angle of incidence. The solid and dotted lines show respectively the horizontal and vertical amplifications, while the points represent the results with the (semi-) analytical method taken from Kawase (1988). As the edges of the canyon are not smooth (discontinuous in their spatial derivatives), some influence does remain, especially outside the canyon in the P-SV case. This occurs because the reflected waves propagate away from the canyon following the given geometry. The same difficulty is encountered even with the discrete wave-number method (Kawase 1988) with respect to Wong's solution $(1979 ; 1982)$. The SH problem is always easier to reproduce by any numerical methods. We observed that a slightly better result is obtained if a partially staggered grid is used for the FDM as opposed to a fully staggered grid. This may be because the partially staggered grid does not require additional interpolations. However, this difference remains minor.

Further numerical tests are carried out through the comparison with a spectral element method (code EFISPEC2D, De Martin 2011). The simulation of PSV-wave propagation inside a hill is performed (Figure 4). The height of the hill 
is normalized to 1 and the ratio of hill-height to length is 0.3 . Thus the P-wave velocity is 1.732 , the $\mathrm{S}$-wave velocity is 1 and the density is 1 . At the lower boundary of the medium, a horizontal displacement is imposed for which the time function is given in Equation (2),

$u(t)=\frac{1}{2} \sqrt{\pi}\left(\frac{\pi^{2}\left(t-t_{s}\right)^{2}}{t_{p}{ }^{2}}-\frac{1}{2}\right) \exp \left(-\frac{\pi^{2}\left(t-t_{s}\right)^{2}}{t_{p}{ }^{2}}\right)$

with $t_{s}=2$ and $t_{p}=1$. The size of the finite difference grid spacing is 0.05 . The time step is 0.0005 . The finite element mesh is composed of 5704 nodes and 9912 elements (950 square elements in the FD-FE interface and 8962 triangular elements in the FE unstructured mesh). It corresponds to about 20 nodes per wavelength for a frequency of $1 / t_{p}$. The boundaries of the finite difference domain are set far enough away from the canyon to avoid artificial reflections. The wave angle of incidence is $\alpha=0^{\circ}$ (horizontal motion only). The ratio of the amplitude of the frequency response on the free surface and the incident wave amplitude at main frequency $f_{0}=1 / t_{p}=1$ is computed for the hybrid method and the spectral element method. Figure 5 shows the displacement amplification at main frequency $f_{0}$ versus the horizontal position. The top and bottom panels correspond respectively to the horizontal and vertical components, normalized by the horizontal amplitude for the flat free-surface case. It should be noted that no vertical component exists in the case of flat free surface. The solid line represents the results with EFISPEC2D, while the dotted line represents the results with the hybrid method. Good agreement is observed between the two different numerical methods, thereby validating the implementation carried out in this study. 


\section{Application: the interaction of topographic irregularities}

\section{Model setting}

As explained in the previous section, a single topographic irregularity (a canyon or a hill) has been studied by many researchers, and many realistic applications are also found. Nevertheless, to the best of our knowledge, few studies deal with the interaction of topographic irregularities. This section aims to ascertain how a series of hills interact as compared to a single irregularity (a hill). Figure 6 shows the model geometry and Table 1 summarizes the model parameters. Henceforth, for the purposes of our discussion, we are designating the hills No. 1 to 7 from left to right. The central hill, No. 4, is located at the middle of the model, i.e., distance $0 \mathrm{~km}$. Each hill is 400 metres long and 200 metres high (see Figure 6), so that the hill length is initially much smaller than the wavelength of around 2100 metres. The series is composed of seven identical hills, with the same geometry. The total length of the topographic irregularity is thus 2800 metres. Although our focus is on a series of irregularities, for the sake of comparison, we also examine the case of a single hill.

The earthquake source is given as a $1250 \mathrm{~m}$-long finite fault in the FDM domain. The source position varies to allow us to study the effect of the angle of incidence on the amplification factor. The source mechanism is given by $\operatorname{dip} \delta=$ $45^{\circ}$ and rake $\lambda=90^{\circ}$ (reverse faulting) on the 2D plane considered (see Figure 6).

\section{Simulation results}

Figure 7 shows snapshots of the wave propagation, magnified around the surface topography, for the case where the source is located $5 \mathrm{~km}$ to the left of the 
centre of the model and at a depth of $5 \mathrm{~km}$. At time step $2.5 \mathrm{~s}$, we can observe the P-wave arrival at the level of the hills and at time step $3.5 \mathrm{~s}$ the S-wave arrival. Then, the S-wave is trapped inside the hills by the reflections along the edges of the hills from time step $4.5 \mathrm{~s}$ onwards. The wave field is deformed by the reflections even outside the hills (time step $6.5 \mathrm{~s}$ ). Figure 8 shows the velocity waveforms along the ground surface for the horizontal (top) and vertical (bottom) components. The abscissa represents time and the ordinate, the distance from the centre of the topographical model. We can identify the P and S-wave arrivals and a reflected $\mathrm{S}$-wave (in the negative direction). The ground motion remains significantly high well after the S-wave arrival between -1400 and 1400 metres, where the topography is irregular.

To quantify the interaction of topographic irregularities, we have selected peak ground velocity (PGV) at the surface for our discussion, one of the most important parameters in earthquake engineering. First Figure 9 shows PGV values for different situations. The first three panels represent the effect of the hill location (a single hill corresponding to the positions of Nos. 1, 4 or 7, cf. Figure 6) with respect to the source, then, for the sake of comparison, the case where there is no topography (a flat free surface), as a reference ground motion, and finally the series of seven hills. First of all, we note that every peak of PGV corresponds to each hill, except for the third example (single hill location at No. $7=6.2 \mathrm{~km}$ from the source), where the hill is located around the node of the seismic wave radiation pattern.

Moreover, the hills close to the source (towards No. 1) seem to have a higher PGV than the hills far from the source (towards No. 7). However, when we look at the ratio of PGVs in the topographic free surface case and PGVs in the flat free surface case (Figure 10), we note that the amplification due to the topography 
is lower for the hills located close to the source than for the hills located far from the source. Indeed, the PGV ratio is lower for the hills nearer from the source than for the hills farther from the source in this particular geometrical configuration, contrary to what would naturally be expected. We also note a motion deamplification at the right-hand side of the farthest hill from the source (No. 7). However, no de-amplification occurs at the trough between the hills. In the case of the series of hills, the PGVs on top the hill farthest to the left (No. 1), the one in the centre (No. 4) and the one farthest right (No. 7) are 0.123, 0.107 and 0.065, respectively, whereas in the cases of the single hill in locations corresponding to the former case, the PGVs are $0.127,0.085$ and 0.035 respectively. The motion amplification due to the neighbouring topography is thus very different depending on the geometrical configuration. When there is no topographic irregularity between the source and the site considered, as in the case of the hill farthest to the left (No. 1), the ground motion amplification is negligible. However, when the topographic irregularity (hills) lies between the pass, as in the case of the hill farthest to the right (No. 7), the motion amplification increases (about twice in this example).

\section{The influence of source location (incident wave direction)}

The effects of the distance of the hills from the source, of the ridge steepness and of the ratio between wavelength and the lengths of the hills are investigated. All sets of parameters are given in Table 2.

In the first set of parameters, the distance between the source and the centre of the topography varies: the source is located successively at 2,5 and 10 $\mathrm{km}$ to the left of the centre of the topography. The focal depth remains at $5 \mathrm{~km}$. Figure 11 shows horizontal PGV versus distance from the centre of the 
topography for the single hill located at the centre of the model (top panels) and the series of hills (bottom panels). The hill closest to the source is not necessarily the one that has the highest PGV on top, due to the directivity of the extended fault source.

Figure 12 shows the ratio of horizontal PGV with and without the topography. We can observe that the ratio for the series of hills is higher than for the single hill. In both topographic configurations, the PGV amplification is highest for the source located $5 \mathrm{~km}$ from the centre of the topography. Indeed, as the dip of the finite fault is $45^{\circ}$, the hill is then located in the rupture direction. In the case of the source located $2 \mathrm{~km}$ from the centre, for the hills nearer the source, the wave incidence is nearly vertical and the PGV ratio is rather low, whereas for the farther hills, the wave incidence angle is higher, inducing a higher PGV ratio due to reflections on the edges of the hills. This phenomenon does not appear for the case with the farthest source.

Finally, Figure 13 shows ground velocity amplitude at frequencies of 0.5 $\mathrm{Hz}$ (top panels) and $1.5 \mathrm{~Hz}$ (bottom panels), still for the same calculations. For the low frequency, each peak corresponds to the top of one hill, but the variation is rather small compared to the ground-motion tendency over a large scale. For the higher frequency, we observe a sharper response corresponding to the hills together with a de-amplification for some of the hills. The hills affected change according to the source distance.

\section{The influence of hill geometry}

In the second set of parameters, the length (L) of the hills is unchanged $(400 \mathrm{~m})$ but the height $(\mathrm{h})$ is set successively at 150,200 and $300 \mathrm{~m}$, corresponding to height-to-length ratios of $0.375,0.5$ and 0.75 . The source is 
located $5 \mathrm{~km}$ to the left of the centre of the topography and at a depth of $5 \mathrm{~km}$. Figure 14 shows horizontal PGV for the single hill located at the centre of the model (top panels) and for the series of hills (bottom panels) for the three heightto-length ratios. In all cases, the spatial variation in PGV is similar, that is, on top of the hills closer to the source, the PGV is higher. Figure 15 shows the ratio of horizontal PGV with and without the topography. Once again, it is found that the ratio for the series of hills is higher than for the case of the single hill. For both topographies, the ratio is amplified when hill height increases. The PGV ratio is lower for the hills nearer the source than for those farther away for this particular source location, contrary to what would naturally be expected. Finally, Figure 16 shows ground velocity amplitude at frequencies of $0.5 \mathrm{~Hz}$ (top) and $1.5 \mathrm{~Hz}$ (bottom).

\section{The influence of incident seismic wavelength}

It is known that the frequency content of the input motion strongly affects site amplification. While it is considered that topographic effects are negligible for long wavelengths, the effects are highest for wavelengths near the horizontal length of the topography. In this study, the length of a single hill is smaller than the wavelength; however, the size of the total topography comprising the seven hills is comparable to the wavelength. We made the ratio between wavelength and the lengths (L) of the hills vary (third model set in Table 2). The finite fault source mechanism, the medium parameters and the hill shapes remain unchanged, but the length of the hills is set successively to 200,400 and $800 \mathrm{~m}$ while the source depth and distance to the left of the centre of the topography are set to $2.5,5$ and $10 \mathrm{~km}$, in order to preserve the proportional geometry with respect to the given frequency. The characteristic source frequency equals the inverse of the duration 
of the source time history function, i.e., $1 \mathrm{~Hz}$. The characteristic wavelength is thus the S-wave velocity (c) multiplied by the source period (T), that is, equal to 2100 metres. The ratio of the lengths of the hills over this wavelength is thus successively $0.095,0.19$ and 0.38 . Figure 17 shows horizontal PGV for the single hill located at the centre of the model (top panels) and for the series of hills (bottom panels) for the three hill-length/wavelength ratios. In all cases, the PGV on top of the hills closer to the source is higher than the PGV on the other hills. Figure 18 shows ratio of horizontal PGV with and without the topography. As in both previous sets of parameters, the ratio for the series of hills is higher than for the single hill case. The PGV ratio is once again lower for the hills nearer from the source than for the hills farther from the source. Finally, Figure 19 shows ground velocity amplitude at frequencies of $0.5 \mathrm{~Hz}$ (top panels) and $1.5 \mathrm{~Hz}$ (bottom panels). For the largest hills (largest L/cT), we observe a de-amplification as compared to the two other cases.

\section{Discussion}

In all previous parametric studies, we chose a series of seven identical hills. The height of the hill is now constant and set to $100 \mathrm{~m}$, but the lengths of the hills increase from $250 \mathrm{~m}$ to $550 \mathrm{~m}$ by an increment of $50 \mathrm{~m}$ from left to right and from right to left (fourth model set). The source is located $2.5 \mathrm{~km}$ to the left of the centre of the topography and at a depth of $2.5 \mathrm{~km}$. Figure 20 shows horizontal PGV versus distance from the centre of the topography on top. On the left panel, the longest hill $(550 \mathrm{~m}$ long) is located farthest to the right and the shortest hill (250 $\mathrm{m}$ long) farthest to the left, and vice versa on the right panel. On the centre panel, all hills are identical (400 m long). The bottom panels show the ratio of horizontal PGV with and without the topography. In the three configurations, we 
observe a de-amplification for the hills located opposite from the source. For the hills located near the source, there is little difference between the three geometrical configurations.

It is difficult to establish systematic rules about amplification resulting from topography on the basis of this study, as the wave field depends strongly on the given parameters both at earthquake source and local site. The presence of a series of hills can either amplify or de-amplify the PGV as compared to the case of a single hill, depending on the geometrical configuration. Table 3 summarizes the PGV on top of a single hill for each set of parameters, the PGV on top of the hill at the centre of the series of hills and the ratio between these two PGVs. Simulations with the first set of parameters show that when the topography is aligned with the rupture direction, the signal amplification due to the interaction between the hills increases.

Moreover, the hill where the PGV ratio is the highest can be the farthest from the source or in the middle of the topography, depending on the geometrical configuration. No set of parameters gave a maximal PGV ratio on the hill closest to the source for this particular source model. Nevertheless, we can conclude that, for both the single hill and the series of hills, the PGV ratio is amplified when the ratio between height and length of the hill increases.

It is useful to mention some computing aspects for further applications. In the example of the last section, the number of finite difference grid nodes is set to $220 \times 70=15400$ for all sets of parameters. The number of finite element nodes varies, depending on the size of the hills. For the $200 \mathrm{~m}$ high and $400 \mathrm{~m}$ long hills, our mesh is composed of 8077 nodes and 10563 elements. With these parameters, our computations last about two hours on a single CPU, dominated by the finite element calculation. However, we did not try to optimize the code nor the size of 
the meshes. Indeed, we have around 20 grids per wavelength in the FD part of the domain, whereas 5 or 6 grids would be enough to avoid numerical dispersion (Moczo et al. 2000). In the FE part, the number of nodes per wavelength is much higher (see Figure 6), and it would be possible to reduce the number of nodes while maintaining a good accuracy of the computation. For further applications, a key task should be optimizing the finite element meshing and its solver. As the finite element method is powerful not only for the simulation of a complex geometry but also for various nonlinear problems, this numerical approach would be useful.

\section{Conclusion}

A hybrid method combining finite element and $4^{\text {th }}$-order finite difference was developed to model $\mathrm{SH}$ and P-SV seismic wave propagation in a $2 \mathrm{D}$ elastic medium with topographic free surface. Both the classic staggered grid finite difference scheme and the partially staggered grid scheme were tested. The accuracy of the hybrid method was studied by comparison with semi-analytical and numerical methods. To illustrate the method, numerical simulations of seismic wave propagation across a series of hills were carried out and compared to the case of a single hill. Depending on the position of the source in relation to the topography, the ratio between the heights and the lengths of the hills or the ratio between the lengths of the hills and the wavelength, the presence of several hills as opposed to a single hill can increase the amplification effect due to the topography. This study highlights the fact that, when evaluating topographic site effects, surrounding topography must be taken into account in addition to local topography. 


\section{Acknowledgements}

We thank H. Kawase and F. De Martin for communicating to us their calculation results for our verification. The comments from the reviewers and the associate editor were useful for improving our manuscript. This work was supported under a BRGM internal research fund program.

\section{References}

Aoi S, Fujiwara H (1999) 3D finite-difference method using discontinuous grids. Bull Seism Soc Am 89:918-930

Badea L, Ionescu IR, Wolf S (2008) Schwarz method for earthquake source dynamics. J Comp Phys 227:3824-3848

Bielak J, Loukakis K, Hisada Y, Yoshimura C (2003) Domain reduction method for threedimensional earthquake modelling in localized regions. Part I: Theory. Bull Seism Soc Am 93:817-824

De Martin F (2011) Verification of a Spectral-Element Method Code for the Southern California Earthquake Center LOH.3 Viscoelastic Case. Bull Seism Soc Am, 101:2855-2865

De Martin F, Modaressi H, Aochi H (2007) Coupling of FDM and FEM in seismic wave propagation. In: $4^{\text {th }}$ International Conference on Earthquake Geotechnical Engineering, Thessaloniki, Greece, 25-28 June 2007

Fäh D (1992) A hybrid technique for the estimation of strong ground motion in sedimentary basins. Dissertation, Swiss Federal Institute of Technology

Gaffet S, Bouchon M (1989) Effects of two-dimensional topographies using the discrete wavenumber - boundary integral equation method in P-SV cases. J Acoust Soc Am 85:2277-2283 Gatmiri B, Arson C, Nguyen KV (2008) Seismic site effects by an optimized 2D BE/FE method I. Theory, numerical optimization and application to topographical irregularities. Soil Dyn Earthquake Eng 28:632-645

Graves RW (1996) Simulating seismic wave propagation in 3D elastic media using staggered-grid finite differences. Bull Seism Soc Am 86:1091-1106

Griffiths DW, Bollinger GA (1979) The effect of Appalachian Mountain topography on seismic waves. Bull Seism Soc Am 69:1081-1105 
Hartzell SH, Carver DL, King KW (1994) Initial investigation of site and topographic effects at Robinwood Ridge, California. Bull Seism Soc Am 84:1336-1349

Kawase H (1988) Time-domain response of a semi-circular canyon for incident SV, P, and Rayleigh waves calculated by the discrete wavenumber boundary element method. Bull Seism Soc Am 78:1415-1437

Komatitsch D, Martin R (2007) An unsplit convolutional Perfectly Matched Layer improved at grazing incidence for the seismic wave equation. Geophysics 72:SM155-167

Komatitsch D, Vilotte J-P (1998) The spectral element method: An efficient tool to simulate the seismic response of 2D and 3D geological structures. Bull Seism Soc Am 88:368-392

Levander AR (1988) Fourth-order finite-difference P-SV seismograms. Geophysics 53:1425-1436

Ma S, Archuleta RJ, Liu P (2004) Hybrid modeling of elastic P-SV wave motion: A combined finite-element and staggered-grid finite-difference approach. Bull Seism Soc Am 94:1557-1563

Madariaga R (1976) Dynamics of an expanding circular fault. Bull Seism Soc Am 65:163-182

Moczo P, Bystrický E, Kristek J, Carcione JM, Bouchon M (1997) Hybrid modeling of P-SV seismic motion at inhomogeneous viscoelastic topographic structures. Bull Seism Soc Am $87: 1305-1323$

Moczo P, Kristek J, Halada L (2000) 3D fourth-order staggered-grid finite-difference schemes: Stability and grid dispersion. Bull Seism Soc Am 90:587-603

Ohminato T, Chouet BA (1997) A free-surface boundary condition for including 3D topography in the finite-difference method. Bull Seism Soc Am 87:494-515

Saenger EH, Gold N, Shapiro SA (2000) Modeling the propagation of elastic waves using a modified finite-difference grid. Wave Motion 31:77-92

Spudich PA, Hellweg M, Lee WHK (1996) Directional topographic site response at Tarzana observed in aftershocks of the 1994 Northridge, California, earthquake: Implications for mainshock motions. Bull Seism Soc Am 86;S193-S208

Trifunac MD (1971) Surface motion of a semi-cylindrical alluvial valley for incident plane SH waves. Bull Seism Soc Am 61:1755-1770

Trifunac MD (1973) Scattering of plane SH waves by a semi-cylindrical canyon. Earthquake Eng Struct Dyn 1:267-281

Virieux J (1986) P-SV wave propagation in heterogeneous media: Velocity-stress finite-difference method. Geophysics 51: 889-901 
Wolf S, Favreau P, Ionescu IR (2006) Finite element - finite difference coupling for elastodynamic rupture propagation. In: AGU Fall Meeting, San Francisco, USA, 11-15 December 2006

Wong HL (1979) Diffraction of P, SV, and Rayleigh waves by surface topographies. Report CE79-05, Department of Civil Engineering, University of Southern California, Los Angeles, California

Wong HL (1982) Effect of surface topography on the diffraction of P, SV, and Rayleigh waves. Bull Seism Soc Am 72:1167-1183

Zahradník J, Moczo P (1996) Hybrid seismic modeling based on discrete-wavenumber and finitedifference methods. Pure Appl Geophys 148:21-38 


\section{Figures}

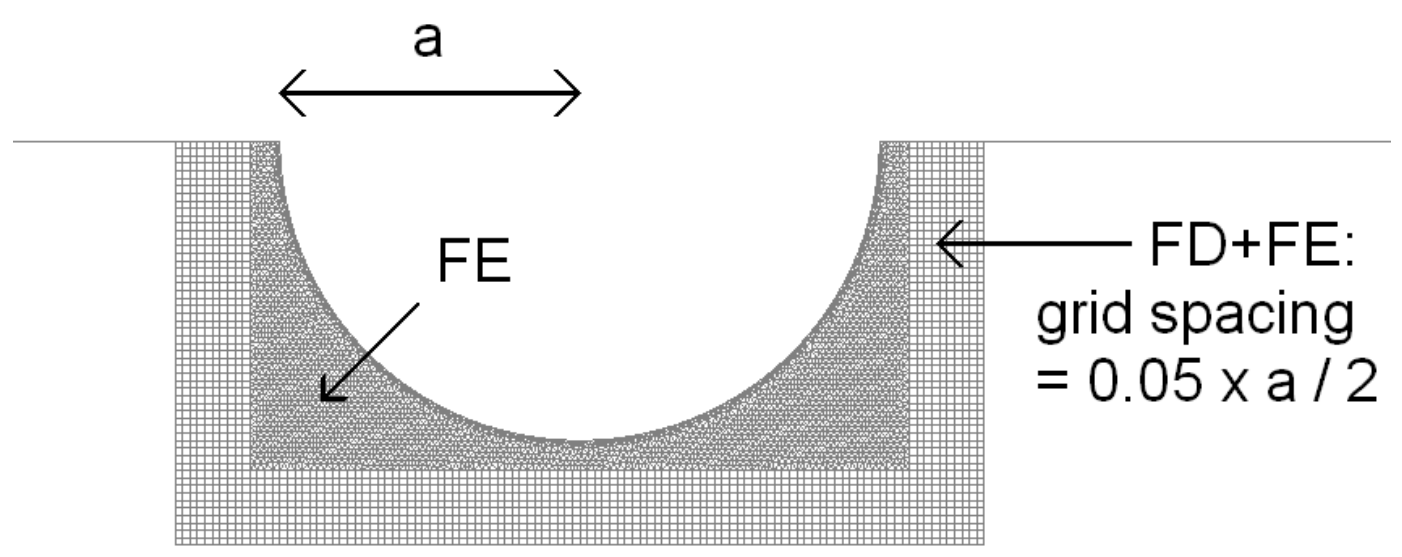

\section{FD: grid spacing $=0.05 \times$ a}

Fig. 1a. Mesh used for the simulation with the fully staggered grid. For the area where both FDM and FEM are computed, the finite-element mesh size is half the finite-difference grid spacing.

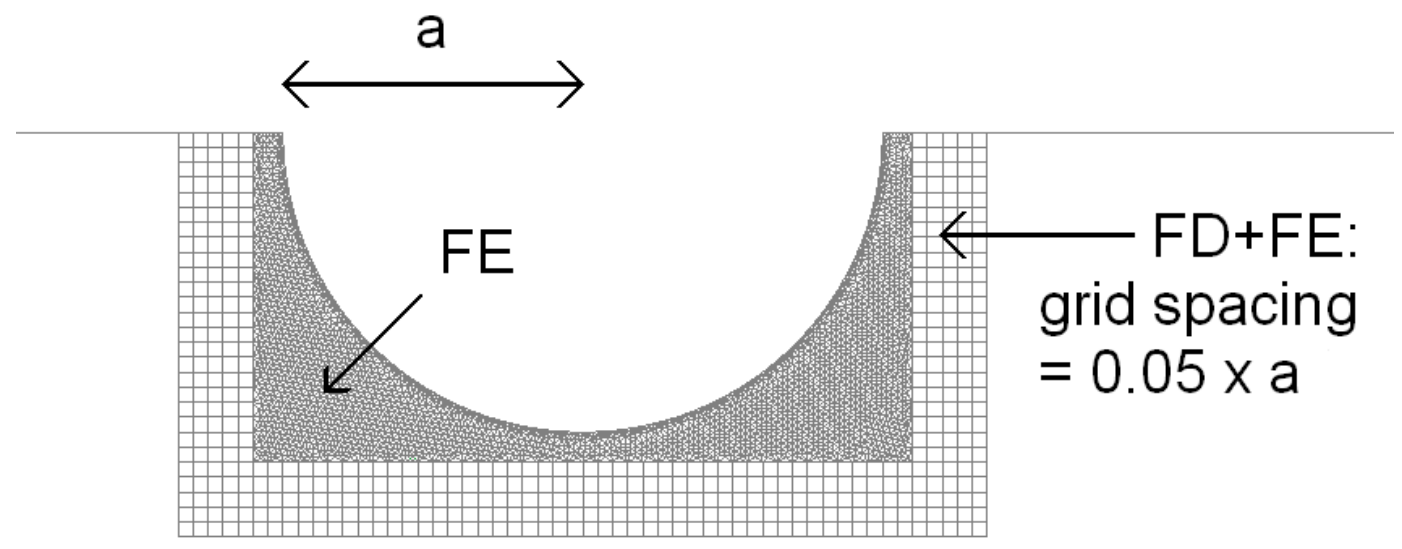

\section{FD: grid spacing $=0.05 \times$ a}

Fig. 1b. Mesh used for the simulation with the partially staggered grid. For the area where both FDM and FEM are computed, the finite-element mesh size is the same as the finite-difference grid spacing. 


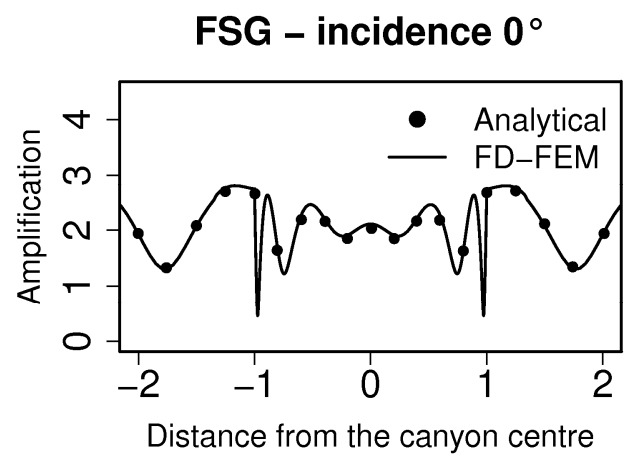

FSG - incidence $30^{\circ}$

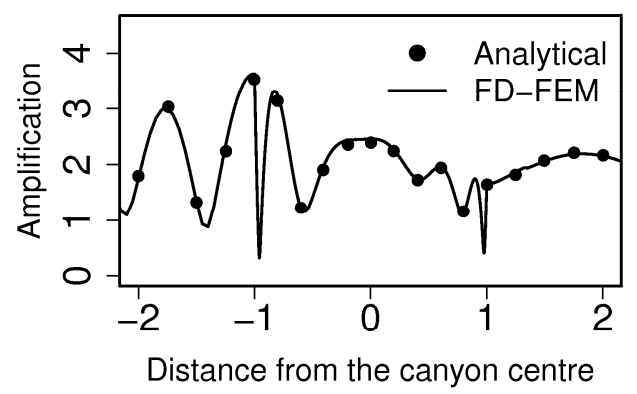

$P S G$ - incidence $0^{\circ}$

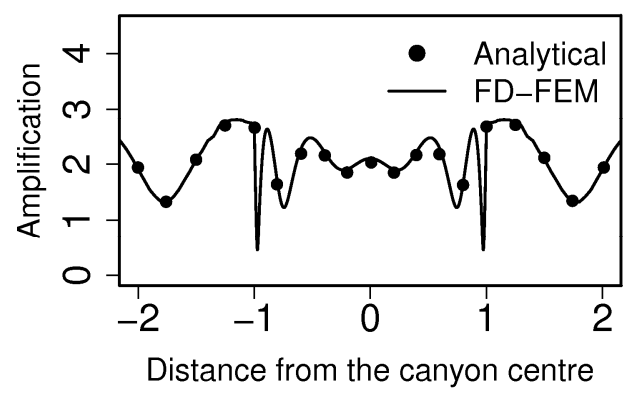

PSG - incidence $30^{\circ}$

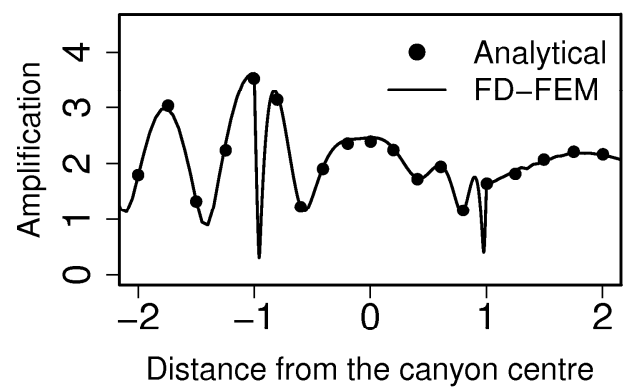

Fig. 2. Displacement amplification of SH waves versus distance from the canyon centre. 


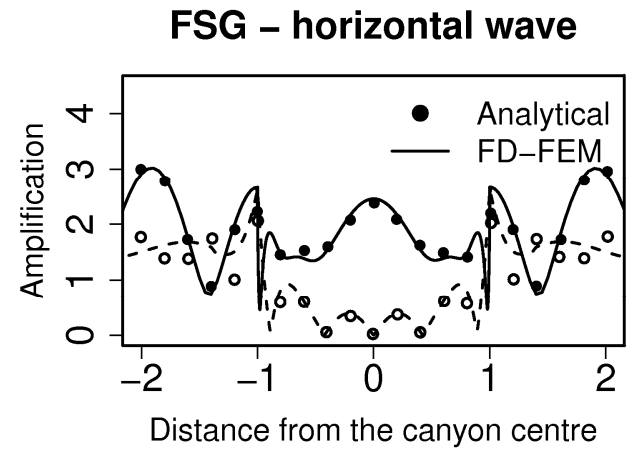

FSG - vertical wave

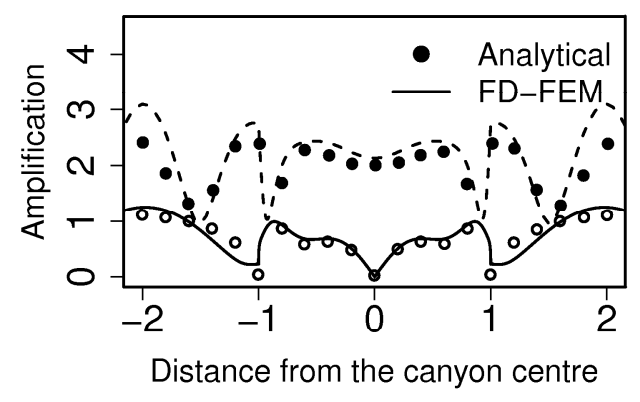

PSG - horizontal wave

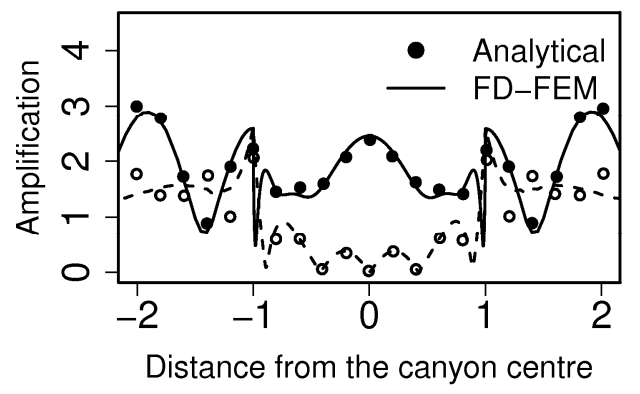

PSG - vertical wave

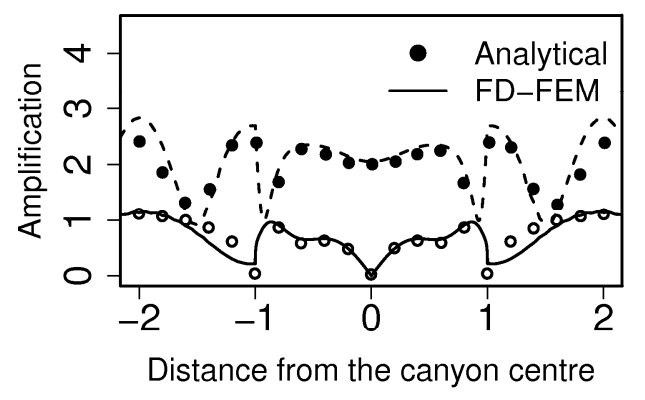

Fig. 3. Displacement amplification of P-SV waves versus distance from the canyon centre. 


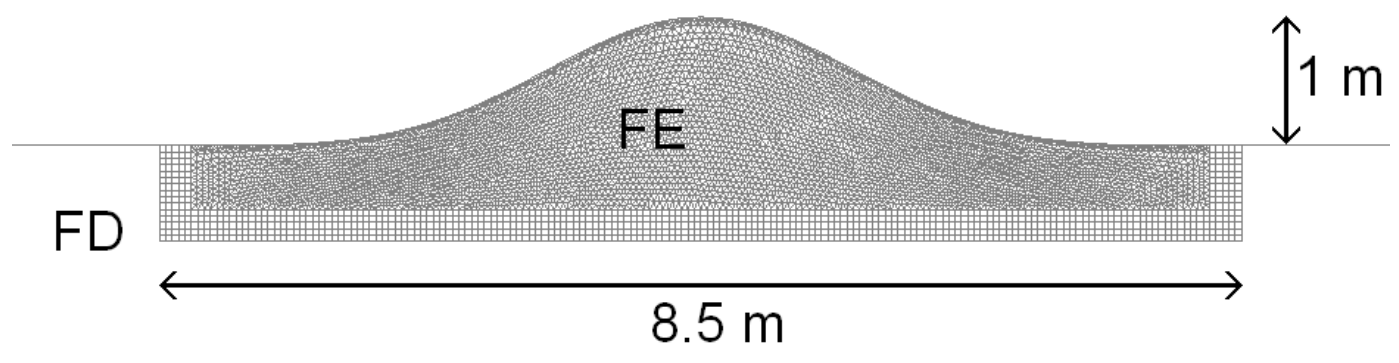

Fig. 4 Mesh used for the comparison with the spectral elements method. 

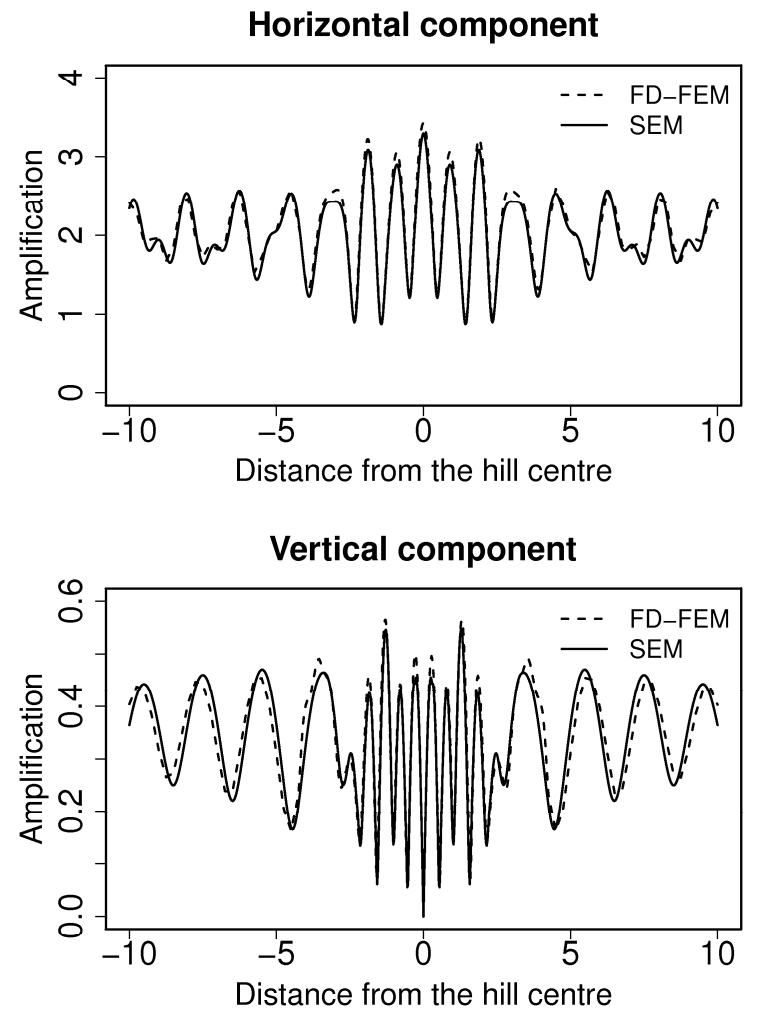

Fig. 5. Frequency response versus the non-dimensional horizontal coordinate. 


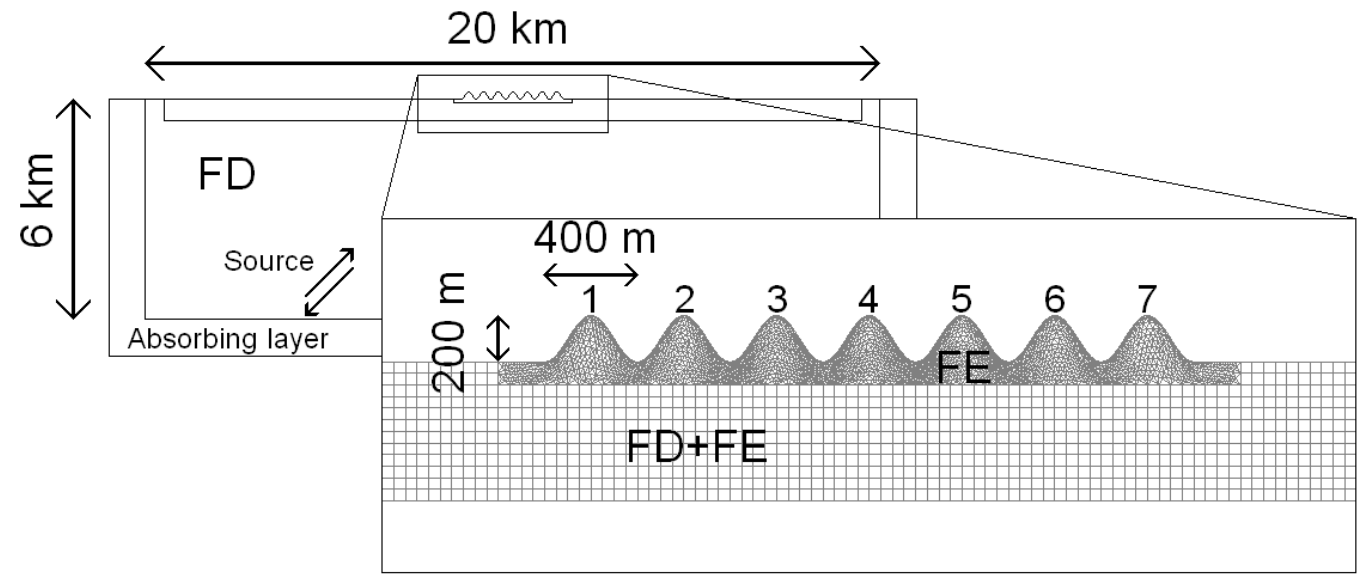

Fig. 6. The geometry of the model composed of a series of seven hills. 


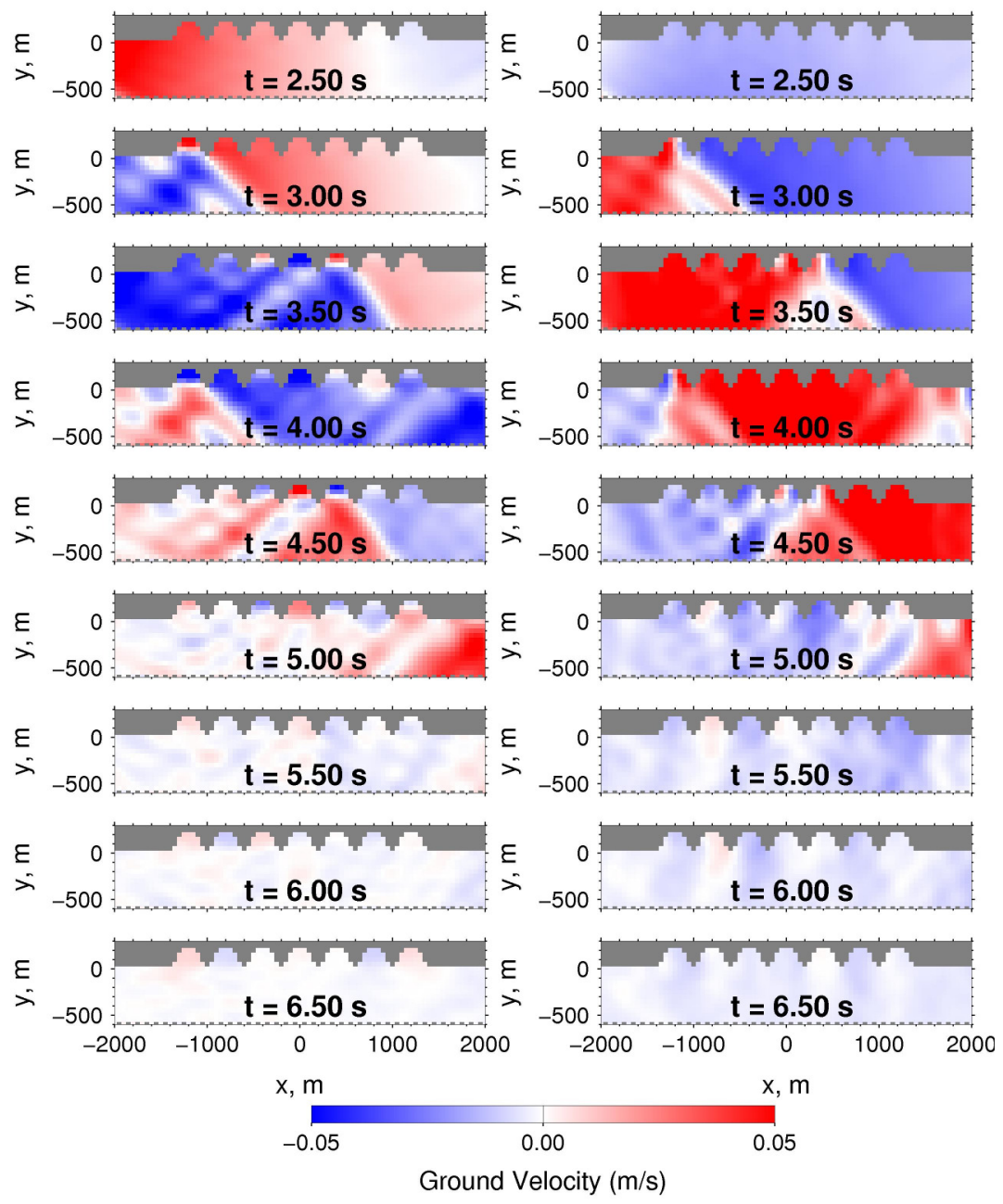

Fig. 7. Snapshots of the seismic wave propagation for the horizontal (left) and vertical (right) components of velocity. 

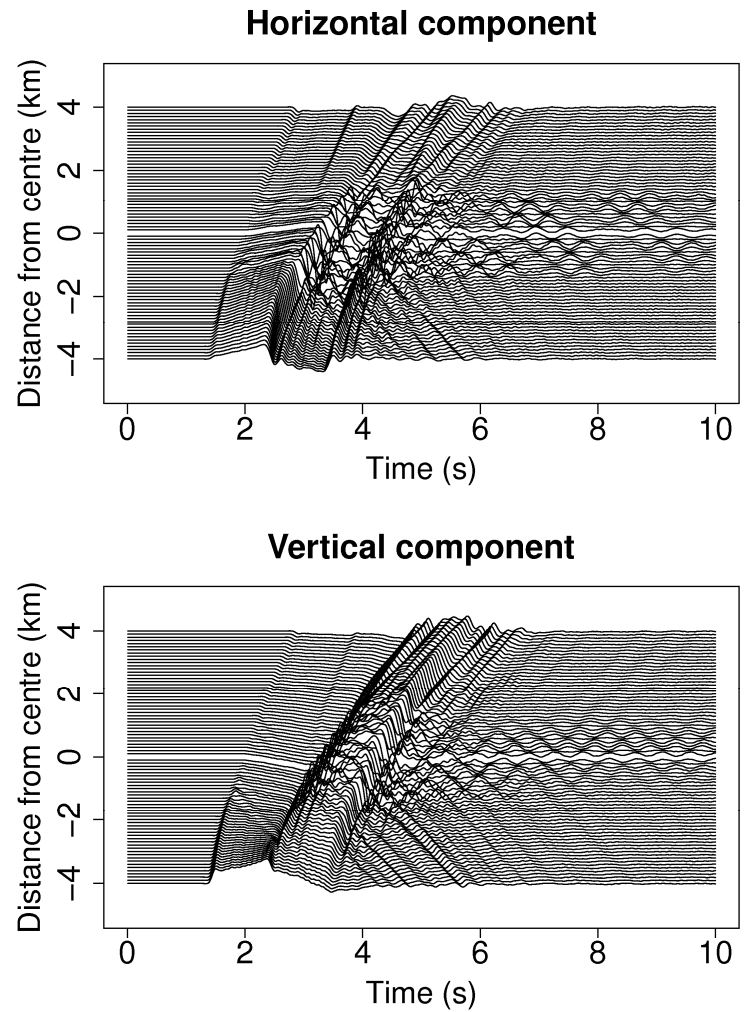

Fig. 8. Synthetic seismograms of the simulation. 

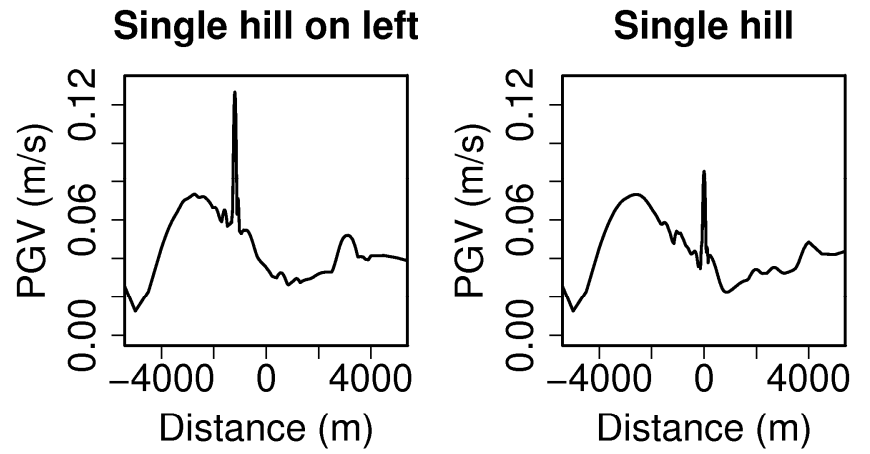

Single hill on right

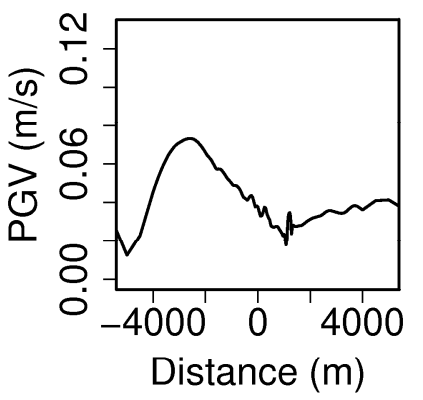

Flat topography
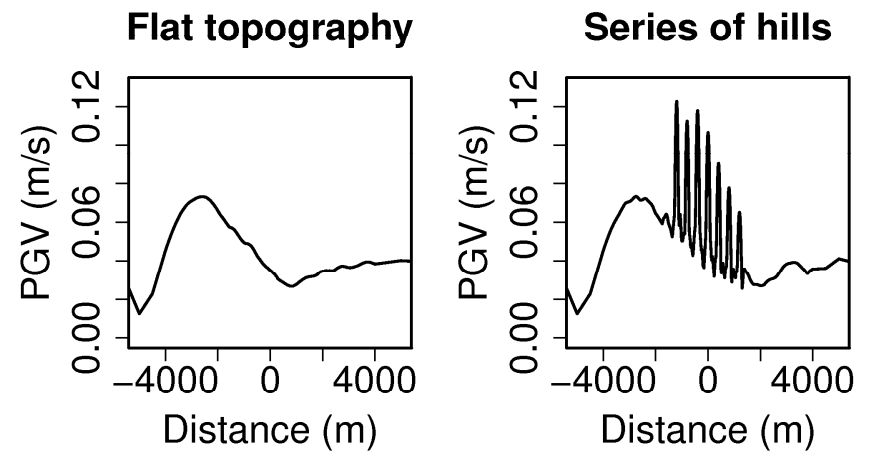

Fig. 9. Horizontal PGV versus distance from the centre of the topography. The three single hills correspond respectively to the positions of Nos. 1, 4 and 7 of the seven-hill case. 

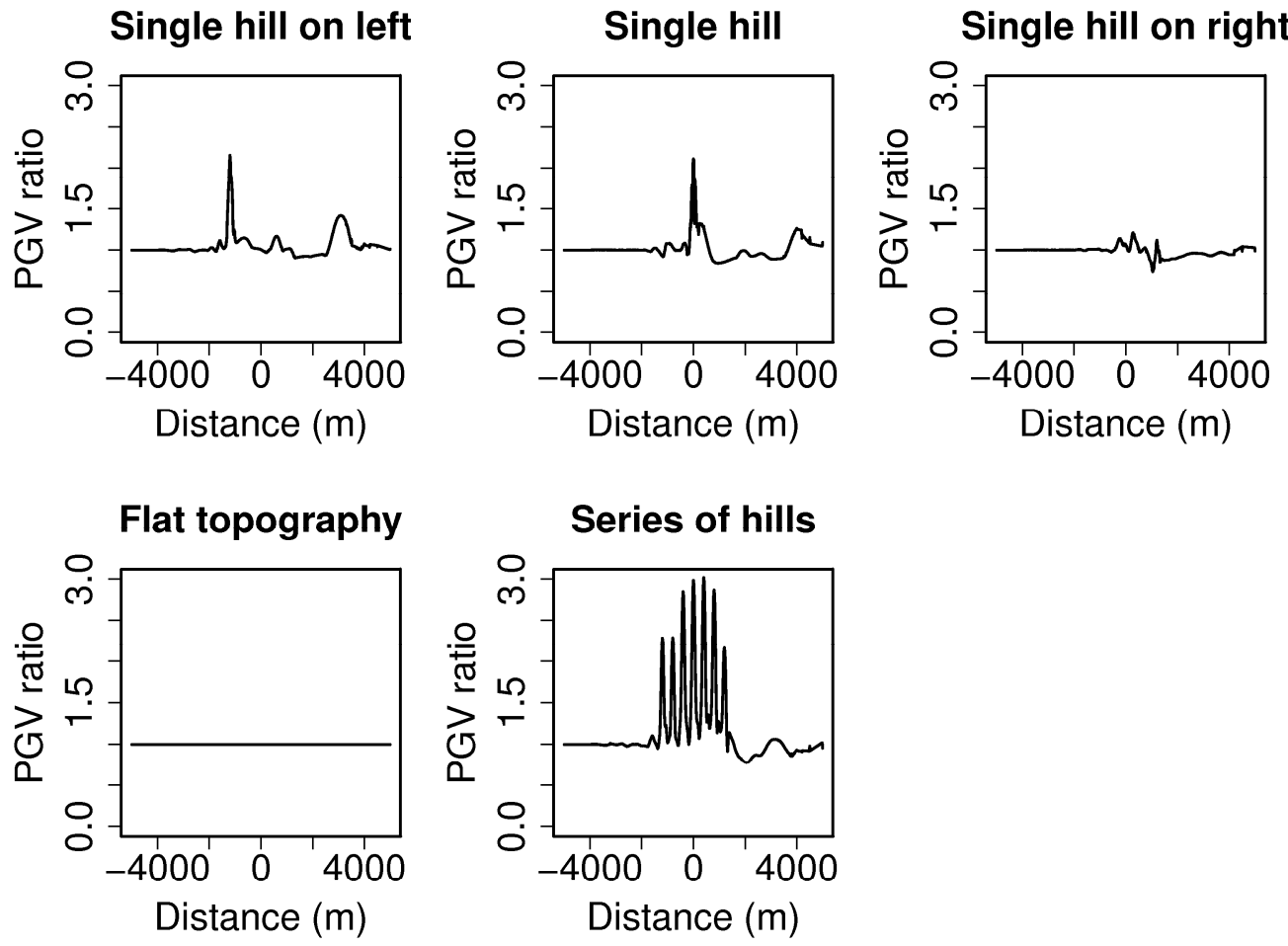

Fig. 10. Ratio of PGV versus distance from the centre of the topography 

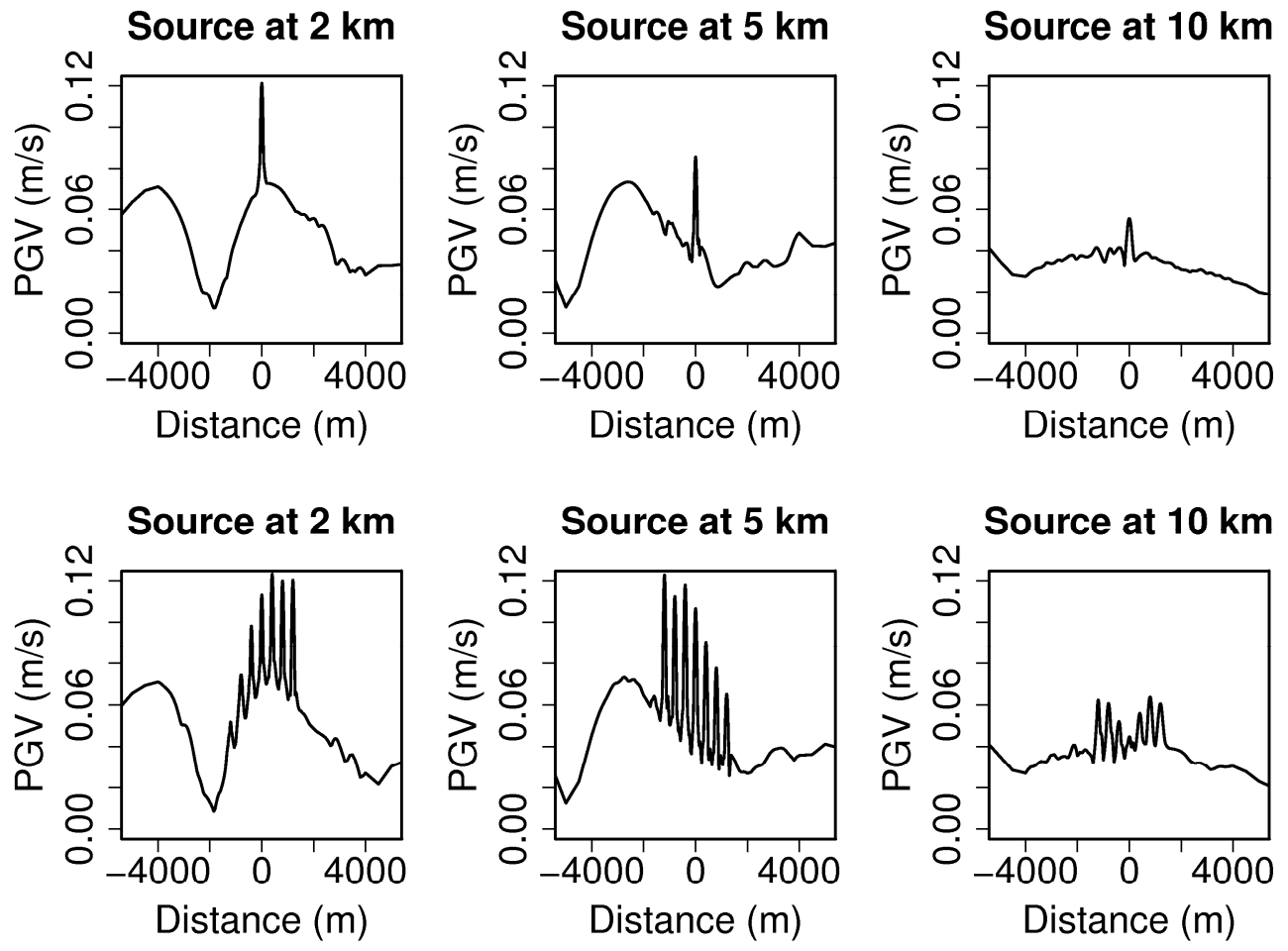

Fig. 11. Horizontal PGV versus distance from the centre of the topography. 

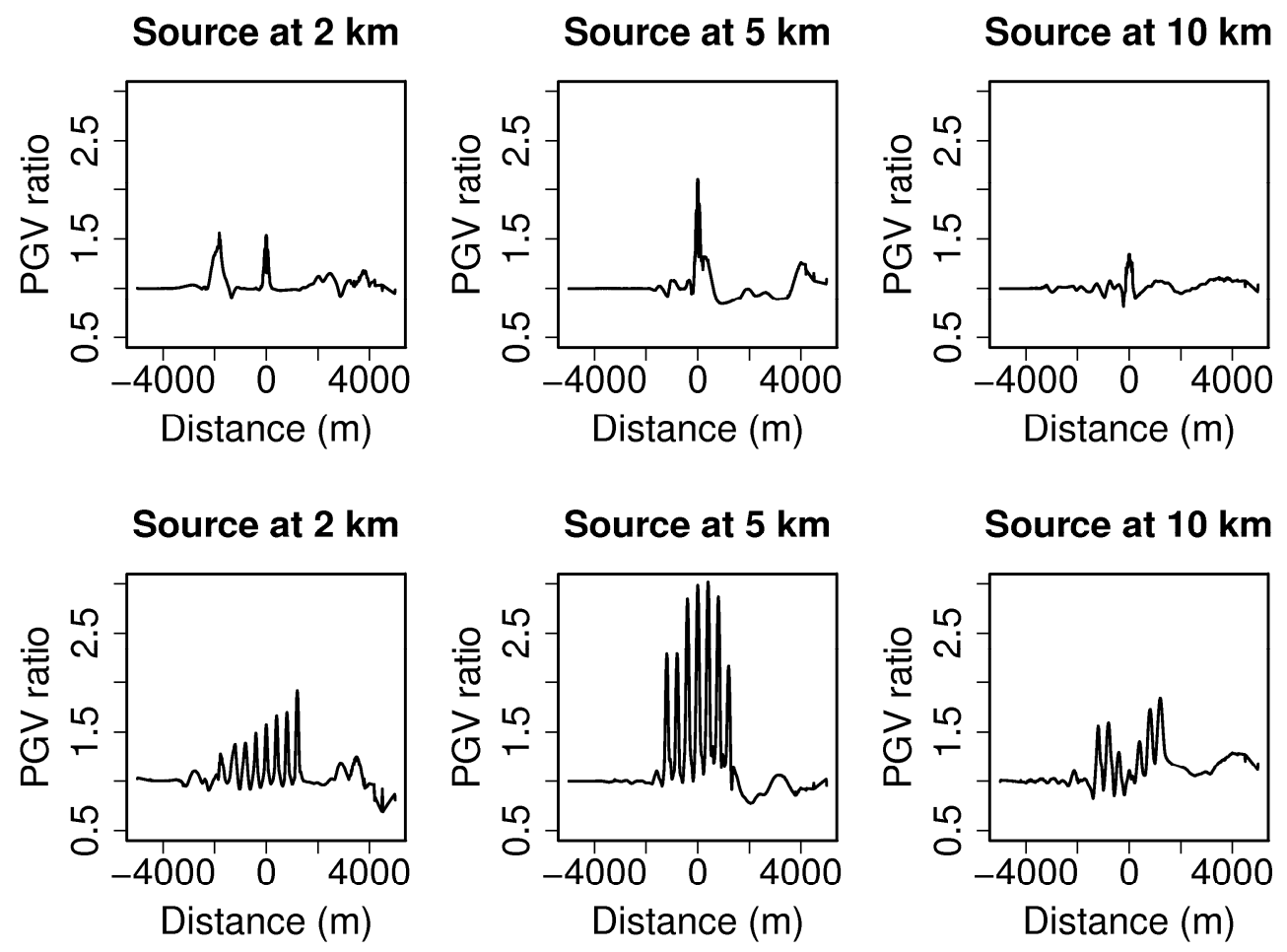

Fig. 12. Ratio of PGV versus distance from the centre of the topography. 

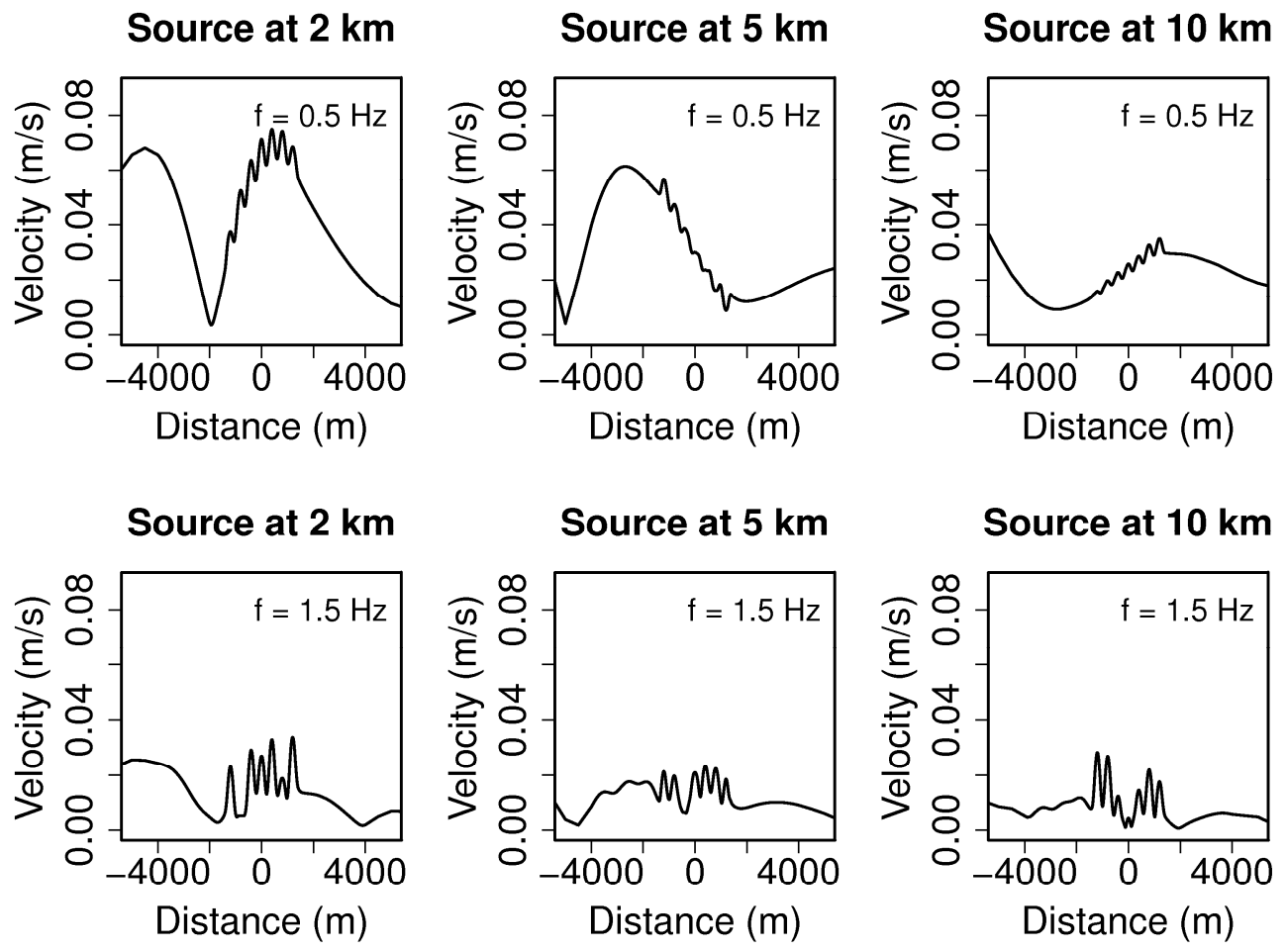

Fig. 13. Ground velocity versus distance from the centre of the topography for selected frequencies. 

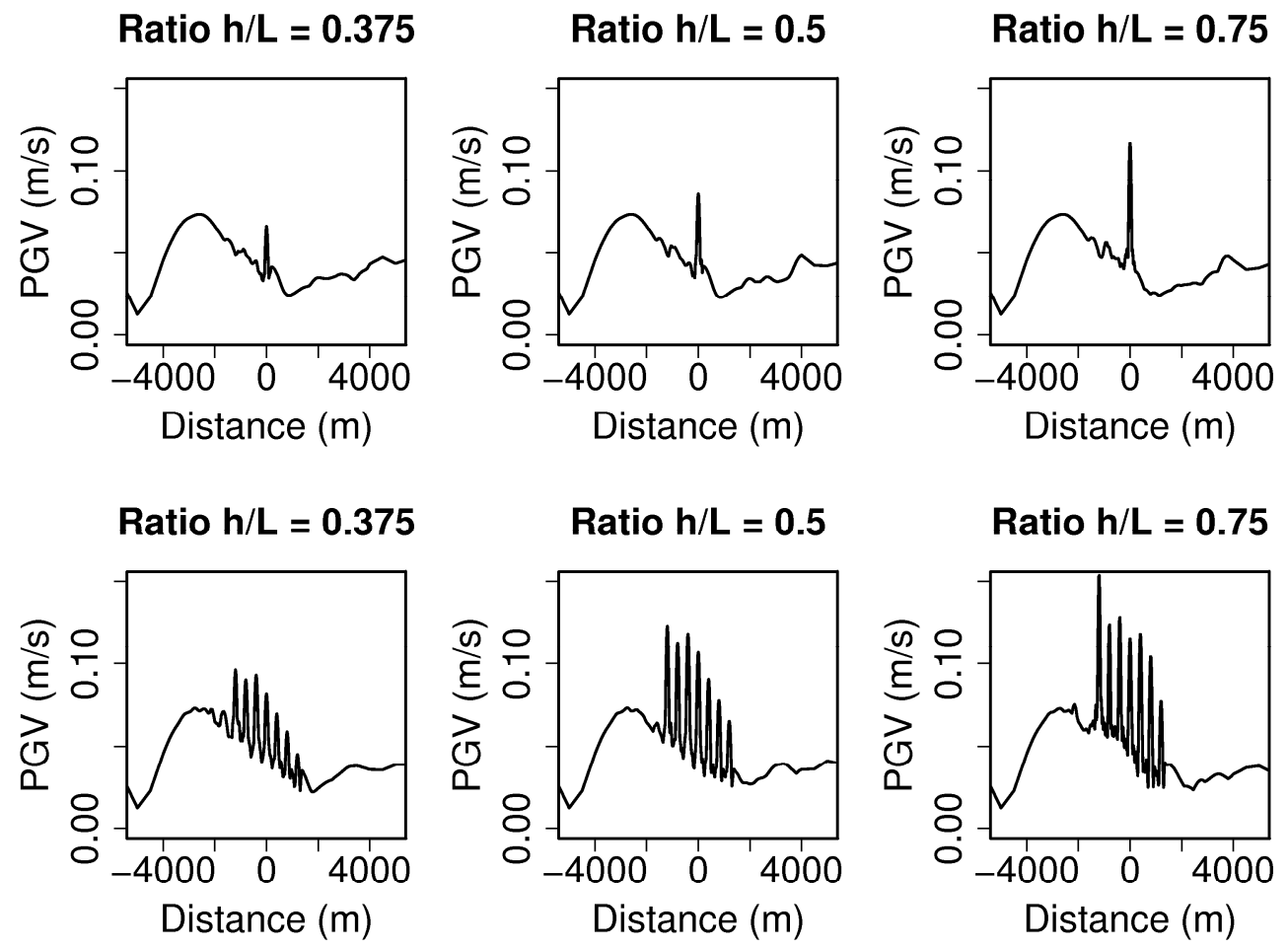

Fig. 14. Horizontal PGV versus distance from the centre of the topography. 

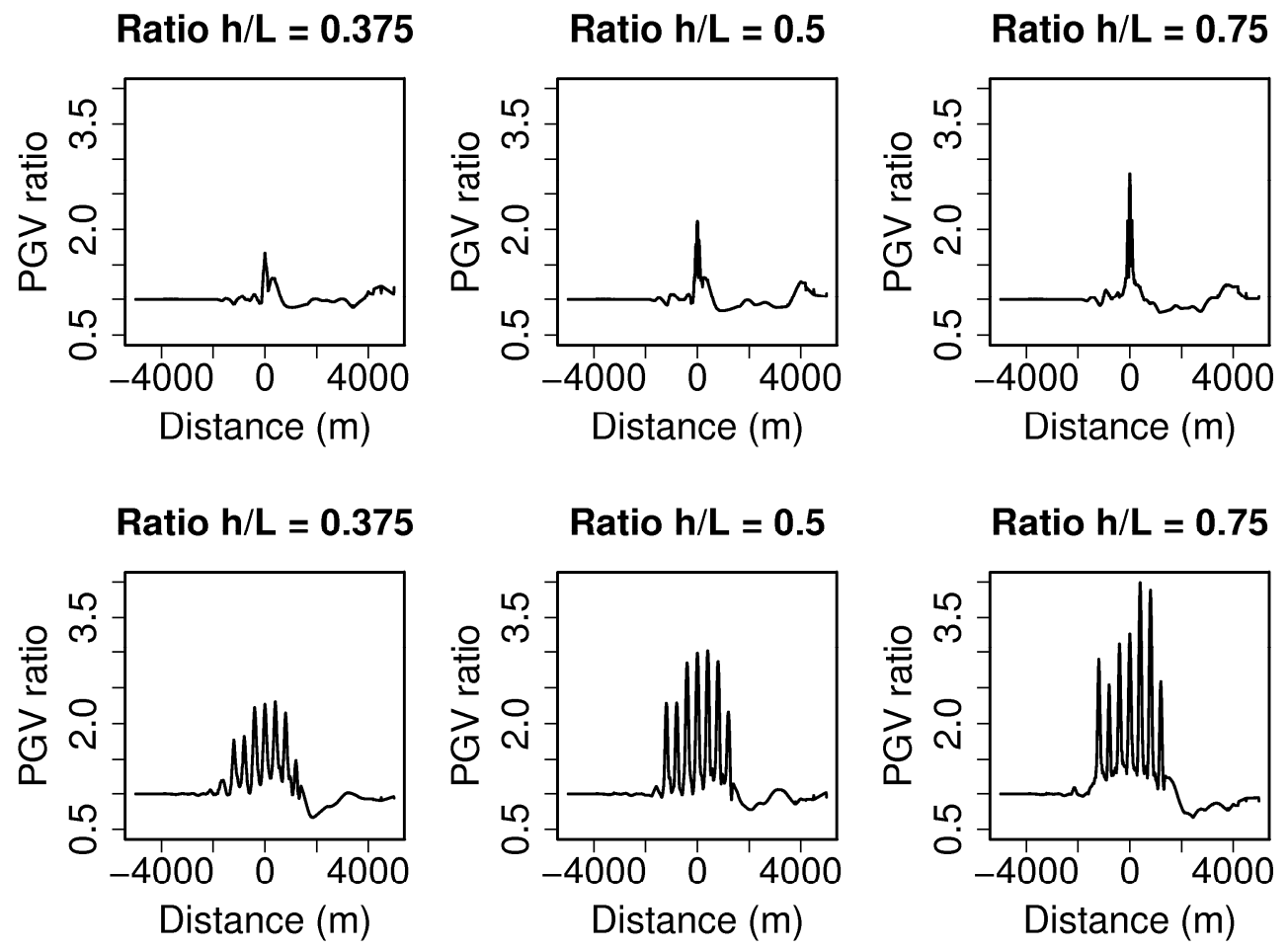

Fig. 15. Ratio of horizontal PGV versus distance from the centre of the topography. 

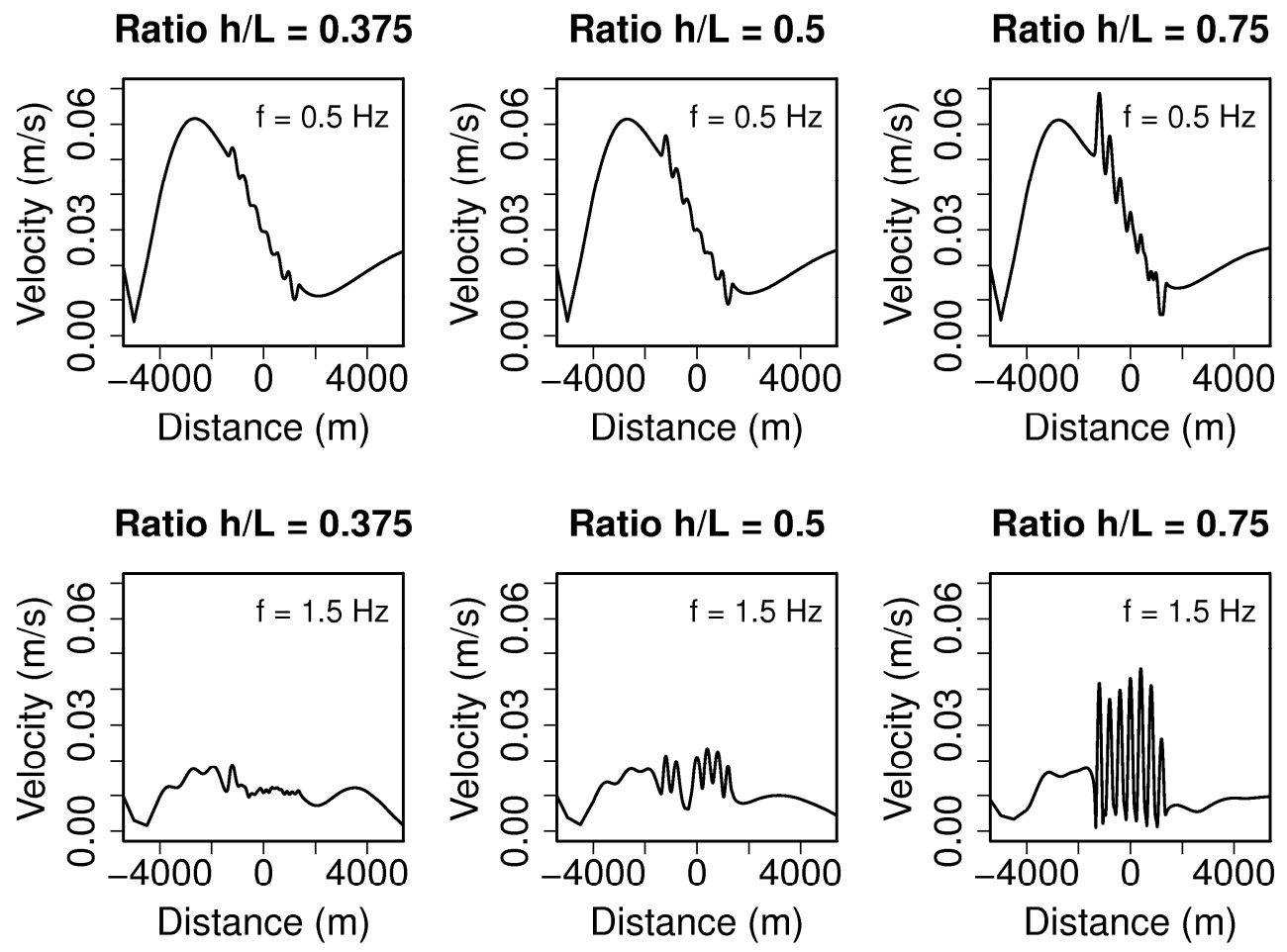

Fig. 16. Ground velocity versus distance from the centre of the topography for selected frequencies. 

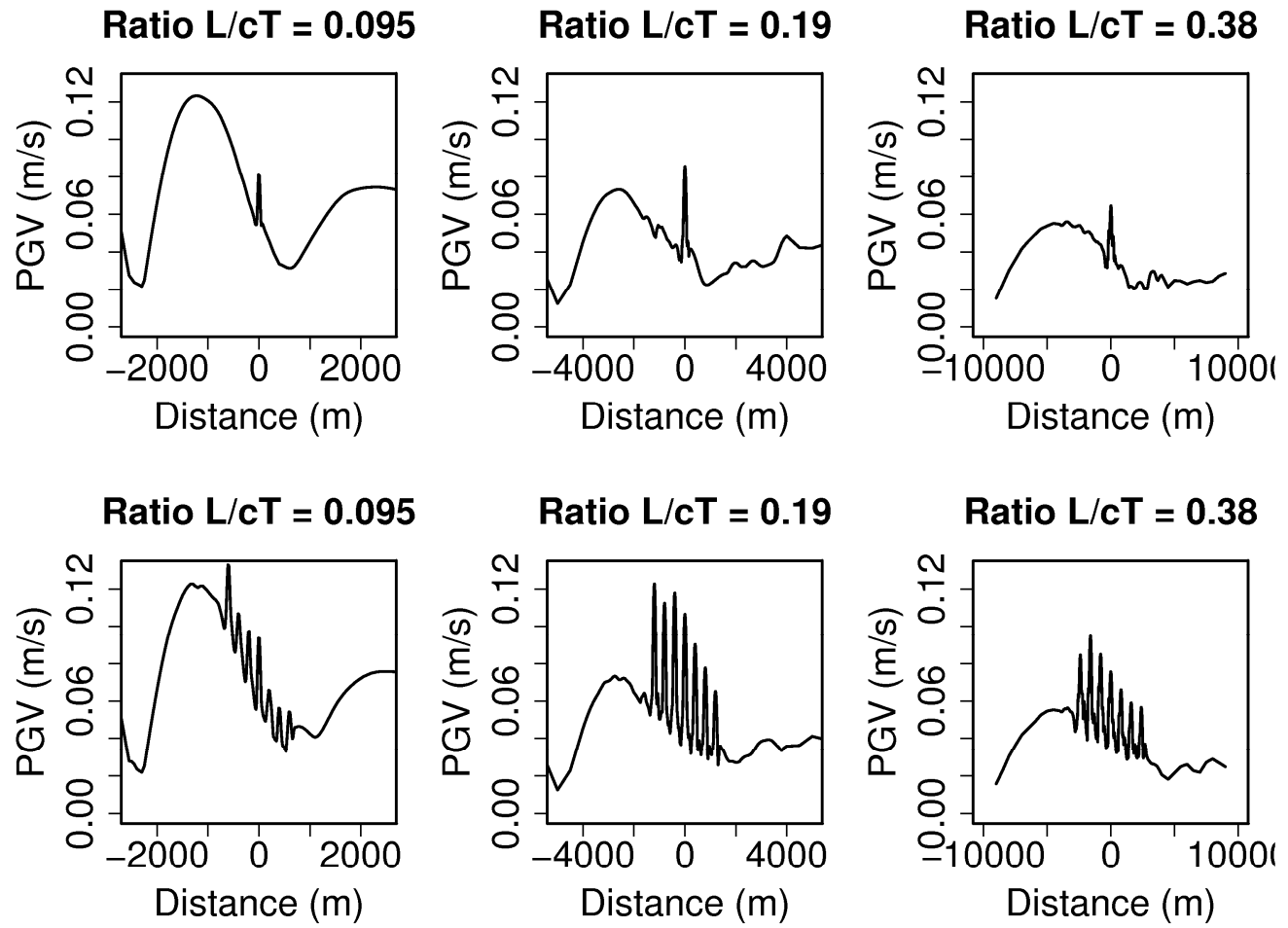

Fig. 17. Horizontal PGV versus distance from the centre of the topography. 

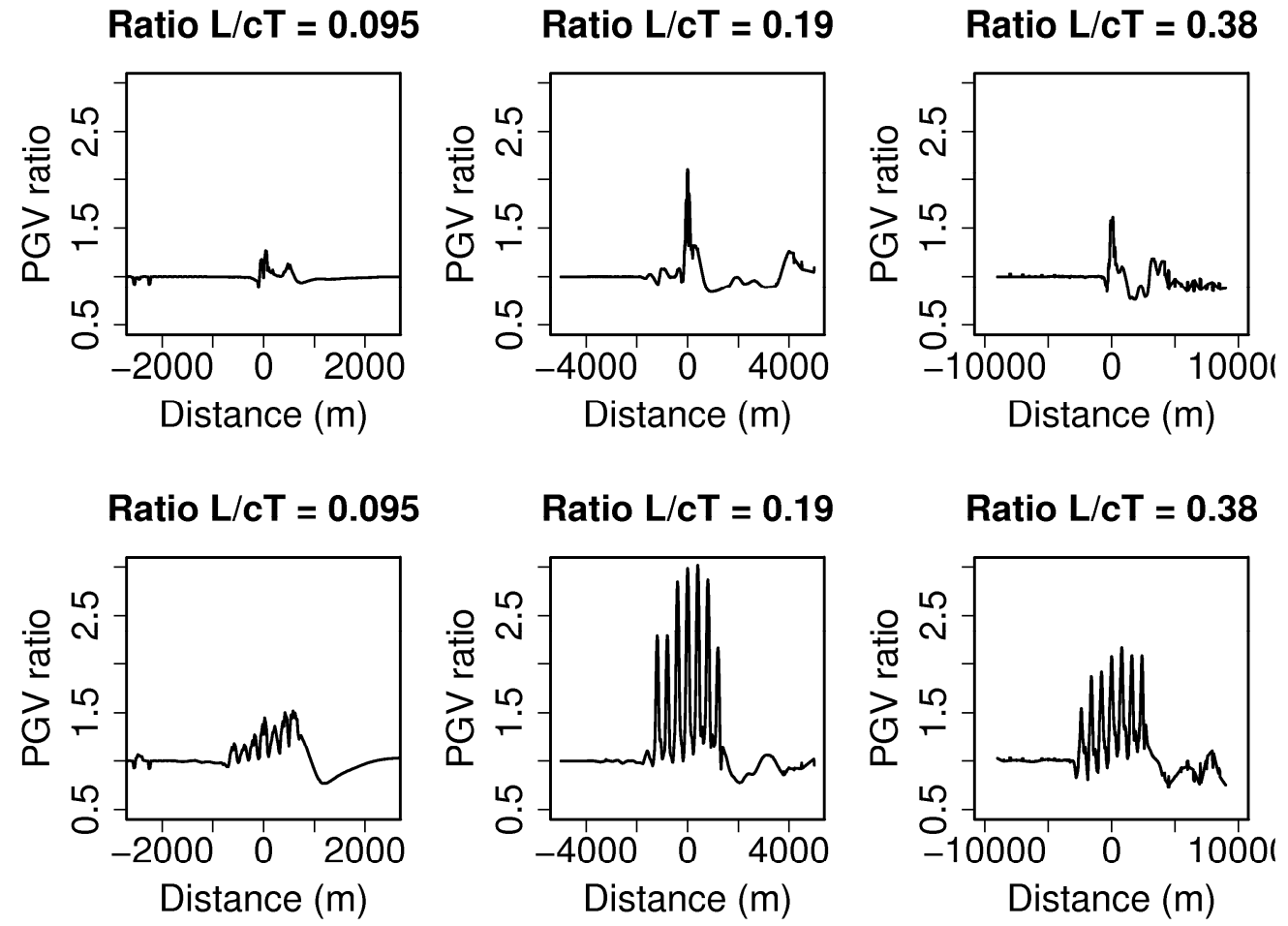

Fig. 18. Ratio of horizontal PGV versus distance from the centre of the topography. 

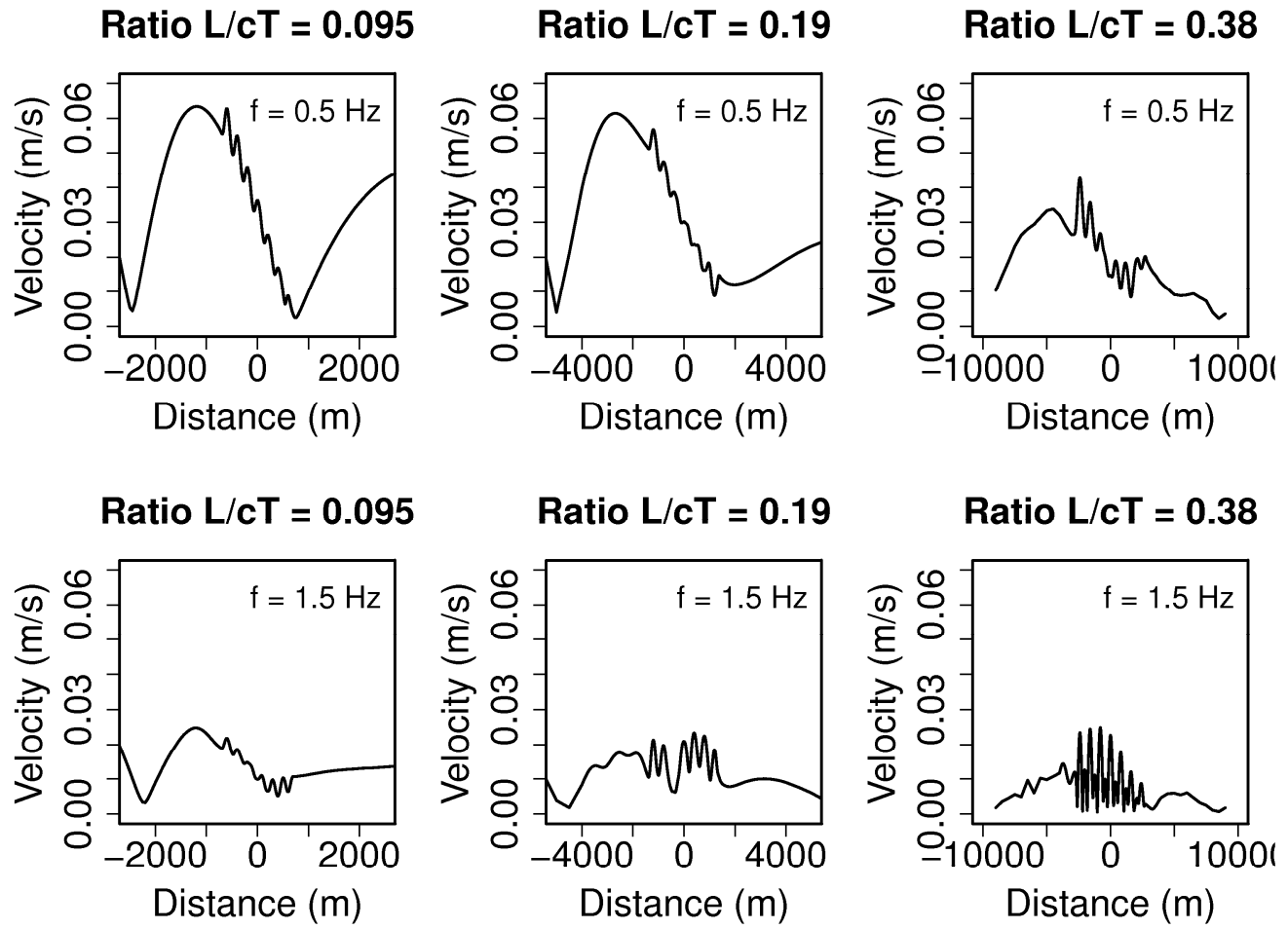

Fig. 19. Ground velocity versus distance from the centre of the topography for selected frequencies. 

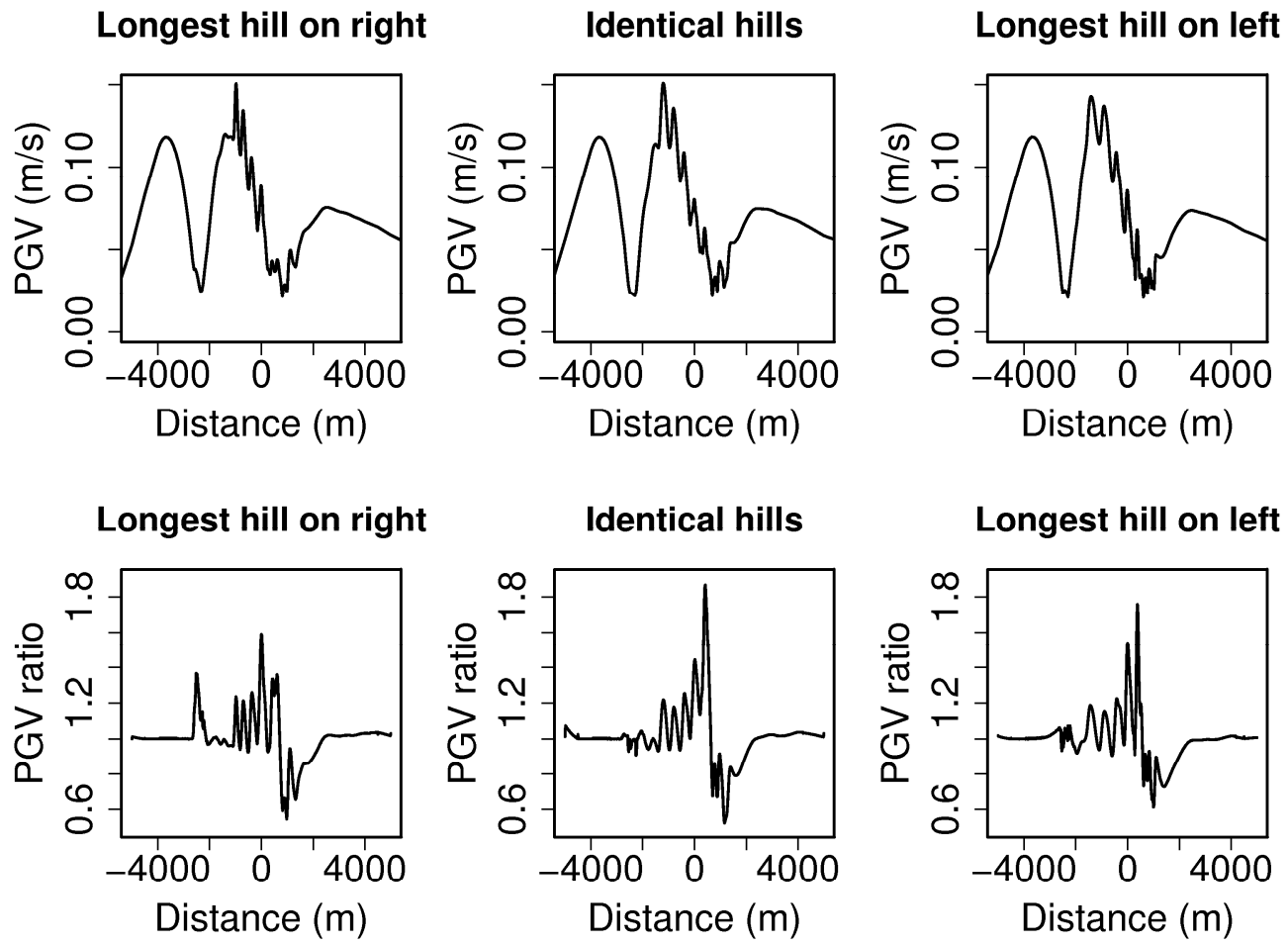

Fig. 20. Horizontal PGV (top) and ratio of horizontal PGV (bottom) versus distance from the centre of the topography. 


\section{Tables}

\begin{tabular}{|c|c|c|}
\hline \multicolumn{2}{|c|}{ Model parameters } & Value \\
\hline \multirow[t]{5}{*}{ Medium parameters } & Width & $20 \mathrm{~km}$ \\
\hline & Height & $6 \mathrm{~km}$ \\
\hline & P-wave velocity & $3637 \mathrm{~m} \cdot \mathrm{s}^{-1}$ \\
\hline & S-wave velocity & $2100 \mathrm{~m} \cdot \mathrm{s}^{-1}$ \\
\hline & Density & $2500 \mathrm{~kg} \cdot \mathrm{m}^{-3}$ \\
\hline \multirow[t]{8}{*}{ Source parameters } & Dip & $45^{\circ}$ \\
\hline & Rake & $90^{\circ}$ \\
\hline & Fault slip & $0.4 \mathrm{~m}$ \\
\hline & Rise time & $1 \mathrm{~s}$ \\
\hline & Rupture velocity & $2500 \mathrm{~m} \cdot \mathrm{s}^{-1}$ \\
\hline & Fault length & $1250 \mathrm{~m}$ \\
\hline & Depth & $5 \mathrm{~km}$ \\
\hline & Distance from centre & $5 \mathrm{~km}$ \\
\hline \multirow[t]{2}{*}{ Computation parameters } & Grid spacing & $100 \mathrm{~m}$ \\
\hline & Time step & $2.510^{-4} \mathrm{~s}$ \\
\hline
\end{tabular}

Tab. 1. Model parameters. 


\begin{tabular}{|c|c|c|c|c|c|}
\hline \multicolumn{2}{|l|}{ Parameters } & Hill length & Hill height & Source depth & Source distance \\
\hline \multirow[t]{3}{*}{ First model set } & 1 & \multirow{3}{*}{$400 \mathrm{~m}$} & \multirow{3}{*}{$200 \mathrm{~m}$} & \multirow{3}{*}{$5 \mathrm{~km}$} & $2 \mathrm{~km}$ \\
\hline & 2 & & & & $5 \mathrm{~km}$ \\
\hline & 3 & & & & $10 \mathrm{~km}$ \\
\hline \multirow[t]{3}{*}{ Second model set } & 1 & \multirow{3}{*}{$400 \mathrm{~m}$} & $150 \mathrm{~m}$ & \multirow{3}{*}{$5 \mathrm{~km}$} & \multirow{3}{*}{$5 \mathrm{~km}$} \\
\hline & 2 & & $200 \mathrm{~m}$ & & \\
\hline & 3 & & $300 \mathrm{~m}$ & & \\
\hline \multirow[t]{3}{*}{ Third model set } & 1 & $200 \mathrm{~m}$ & $100 \mathrm{~m}$ & $2.5 \mathrm{~km}$ & $2.5 \mathrm{~km}$ \\
\hline & 2 & $400 \mathrm{~m}$ & $200 \mathrm{~m}$ & $5 \mathrm{~km}$ & $5 \mathrm{~km}$ \\
\hline & 3 & $800 \mathrm{~m}$ & $400 \mathrm{~m}$ & $10 \mathrm{~km}$ & $10 \mathrm{~km}$ \\
\hline \multirow[t]{3}{*}{ Fourth model set } & 1 & 250 to $550 \mathrm{~m}$ & \multirow{3}{*}{$100 \mathrm{~m}$} & \multirow{3}{*}{$2.5 \mathrm{~km}$} & \multirow{3}{*}{$2.5 \mathrm{~km}$} \\
\hline & 2 & $400 \mathrm{~m}$ & & & \\
\hline & 3 & 550 to $250 \mathrm{~m}$ & & & \\
\hline
\end{tabular}

Tab. 2. Sets of model parameters.

\begin{tabular}{|l|l|l|l|l|l|l|l|l|l|l|}
\hline & \multicolumn{2}{|l}{ First model set } & \multicolumn{2}{l|}{ Second model set } & \multicolumn{3}{l|}{ Third model set } \\
\cline { 2 - 10 } & 1 & 2 & 3 & 1 & 2 & 3 & 1 & 2 & 3 \\
\hline $\begin{array}{l}\text { PGV on top of the single } \\
\text { hill }\end{array}$ & 0.121 & 0.086 & 0.056 & 0.066 & 0.086 & 0.117 & 0.081 & 0.086 & 0.064 \\
\hline $\begin{array}{l}\text { PGV on top of the hill at } \\
\text { the centre of the series of } \\
\text { hills }\end{array}$ & 0.113 & 0.107 & 0.045 & 0.082 & 0.107 & 0.115 & 0.094 & 0.107 & 0.076 \\
\hline Ratio of PGVs & 0.93 & 1.24 & 0.80 & 1.24 & 1.24 & 0.98 & 1.16 & 1.24 & 1.19 \\
\hline
\end{tabular}

Tab. 3. PGV for each set of parameters. 